\title{
Biological studies of radiolabeled glucose analogues iodinated in positions 3, 4 or 6
}

\author{
Pascale Perret ${ }^{1}$, Catherine Ghezzi $^{1}$, Lionel Ogier ${ }^{2}$, Medhi Abbadi ${ }^{2}$, Christophe Morin ${ }^{2}$, Jean-Paul Mathieu ${ }^{1}$, Daniel Fagret ${ }^{1}{ }^{*}$ \\ 1 Radiopharmaceutiques Biocliniques INSERM : E340, Université Joseph Fourier - Grenoble I, Faculte de Medecine 38700 LA \\ TRONCHE,FR \\ 2 LEDSS, Laboratoire d'études dynamiques et structurales de la sélectivité CNRS : UMR5616, Université Joseph Fourier - Grenoble I, bat. \\ Chimie recherche 301 Rue de la Chimie - BP 5338041 GRENOBLE CEDEX 9,FR
}

* Correspondence should be adressed to: Daniel Fagret <DFagret@chu-grenoble.fr >

\begin{abstract}
The aim of this study was to assess the biological behavior of new radiolabeled glucose analogues proposed as tracers of glucose uptake in vivo and iodinated in position 3,4 , or 6 . Biological results obtained in vitro on adipocytes and erythrocytes and in vivo in mice were compared to those obtained with the gold-standard tracer of glucose uptake, 2-deoxy-D-glucose. None of these molecules had the same biological behavior than 2-deoxy-D-glucose. Therefore, these compounds cannot be considered as tracers of glucose uptake.

MESH Keywords Adipocytes ; metabolism ; radionuclide imaging ; Animals ; Cells, Cultured ; Erythrocytes ; metabolism ; radionuclide imaging ; Female ; Fluorodeoxyglucose F18 ; diagnostic use ; pharmacokinetics ; Glucose ; analogs \& derivatives ; diagnostic use ; pharmacokinetics ; Humans ; Iodine Radioisotopes ; diagnostic use ; pharmacokinetics ; Isotope Labeling ; methods ; Male ; Metabolic Clearance Rate ; Mice ; Organ Specificity ; Radiopharmaceuticals ; diagnostic use ; pharmacokinetics ; Rats ; Rats, Wistar ; Tissue Distribution ; Tomography, Emission-Computed, Single-Photon ; methods
\end{abstract}

Author Keywords Iodinated glucose analogues ; Glucose uptake ; RadiopharmaceuticalsSPECT

\section{Introduction}

A number of diseases are associated with glucose transport and metabolism defects, such as myocardial ischemia [1 ], type 2 diabetes [ 2 ], or cancer [3, 4 ]. The development of new radiolabeled glucose analogues for the non-invasive assessment of glucose uptake by nuclear imaging is therefore of great clinical interest. $\left[{ }^{18} \mathrm{~F}\right]$-2-fluoro-2-deoxy-D-glucose (FDG) is the only radiolabeled glucose analogue to be currently used clinically with Positron Emission Tomography (PET) in cardiology [1, 5 ], neurology [6, 7 ], and more recently in oncology [3, 8 ]. However, ${ }^{18} \mathrm{~F}$ is a $\beta+$ emitter with a short half-life $(110 \mathrm{~min})$, requiring a cyclotron in close proximity to the site of tracer utilization. The number of PET centers is increasing but remains limited. The development of a glucose analogue labeled with a $\gamma$-emitter for Single Photon Emission Tomography (SPECT) and readily available for routine use in all Nuclear Medicine departments is therefore still of great clinical interest. ${ }^{123} \mathrm{I}$ is a pure $\mathrm{y}$-emitter with a 13.3 hours half-life and a $123-160 \mathrm{keV}$ photon energy perfectly fitted for SPECT. Several radiolabeled glucose analogues have been proposed so far but none of them demonstrated the same biological behavior as FDG $[9,10,11,12,13,14,15,16,17]$. In this study, the biological behavior of four original glucose analogues iodinated in position 3, 4 and 6 proposed as tracers of glucose uptake in vivo was investigated. A tracer of glucose uptake should enter the cells via the glucose transporters (GLUTs) and accumulate intracellularly without being further metabolized or degraded for a period long enough to allow SPECT imaging. Each tracer should therefore present several performance characteristics such as tissue selectivity, high cellular uptake, good affinity for the GLUTs, strong retention, and good interaction with hexokinase. In the present study, in vivo mouse biodistribution experiments were performed in order to demonstrate a potential accumulation of the iodinated glucose analogues in an organ. Two in vitro models were also used to investigate the cellular uptake mechanisms of the tracers. Human erythrocytes have been widely used to study glucose uptake because glucose transporters are highly expressed within their cellular membranes [18 ]. However, as erythrocytes mainly express the insulin-insensitive GLUT1 transporter, adipocytes were also used as a model for the insulin-sensitive GLUT4 transporter.

\section{Materials and Methods}

\section{Materials}

Cytochalasin B, Glucose (Hexokinase) kit [Method \#16-UV] and common chemical products were obtained from Sigma-Aldrich (Saint Quentin Fallavier, France). Insulin was obtained from Novo Nordisk (Paris, France), BSA (fraction V) from Intergen (Edinburgh, UK) and crude collagenase from Boehringer (Mannheim, Germany). $\left[{ }^{14} \mathrm{C}\right]$-2-deoxy-D-glucose was obtained from ICN (Paris, France) and ${ }^{123} \mathrm{I}$ and ${ }^{125} \mathrm{I}$ were purchased from Cis Bio international (Paris, France). Wistar rats and Swiss mice were obtained from Iffa Credo (Lyon, France).

\section{Synthesis}

3- C -iodomethyl- $\alpha, \beta-D-$ glucopyranoside (3IMG) (Fig. 1a ) was obtained by addition of an iodomethyl group on carbon 3 of the glucose molecule. 4-deoxy-4-iodo-D-glucose (4DIG) (Fig. 1b ) was prepared according to the method of Abbadi et al. [19 ]. 4-O -(2' 
-iodoethyl)- $\alpha, \beta$-D-glucopyranoside (4IEG) (Fig. 1c ) was obtained by grafting the stable $\beta$-iodoethoxyl group to position 4 [20 ]. 6- C -(4' -iodophenyl)-L-glycero- $\alpha, \beta-D$-glucopyranoside (6IPG) (Fig. 1d ) was prepared by addition of an iodophenyl group to position 6 .

\section{Radiolabeling of glucose analogues}

The labeling of 3IMG and 4IEG was performed by ${ }^{125} \mathrm{I}^{127} \mathrm{I}$ isotopic exchange using the method of Mathieu et al. [21 ]. Five milligrams of the unlabeled glucose analogue were dissolved into $1 \mathrm{~mL}$ of a Na ${ }^{127}$ I solution $(2 \mu \mathrm{g} / \mathrm{mL})$ in acetone before being added to a solution of sodium iodide ${ }^{125} \mathrm{I}$ in $0.02 \mathrm{M} \mathrm{NaOH}$. The mixture was heated at $105^{\circ} \mathrm{C}$ for 60 minutes. Following cooling, acetone was eliminated by evaporation under vacuum and the glucose analogue was dissolved into physiologic medium $(\mathrm{NaCl} 0.9 \%)$. After labeling, free iodides typically representing $20-40 \%$ of the total activity were removed by filtration on an anionic resin (AG1-X8 200-400 mesh, Bio Rad). The radiochemical purity was higher than $95 \%$. The radiolabeling of 4DIG was performed using the tetraacetyl precursor with a triflate group in position $4.1 .3 \mathrm{mg}$ of this precursor was dissolved into $1 \mathrm{~mL}$ of a Na ${ }^{127} \mathrm{I}$ solution $(1 \mu \mathrm{g} / \mathrm{mL})$ in acetone. $\mathrm{Na}^{125} \mathrm{I}$ was then added and the mixture was heated at $105^{\circ} \mathrm{C}$ for 30 minutes. Deprotection of the iodinated product was obtained by treatment with methanol and sodium methylate for 1 hour. Neutralization was then performed with sulfuric acid $\left(10^{-3} \mathrm{M}\right)$. The radiochemical purity was $\sim 80 \%$. The radiolabeling of 6IPG was performed using the stannic equivalent of the final molecule (tributyl stain) in which several functions were protected. The active form of iodine was obtained by action of chloramin $\mathrm{T}(1 \mathrm{mg} / \mathrm{mL})$ on radioactive sodium iodide (185 MBq) in the presence of the compound precipitated in a $0.1 \mathrm{M}$ ethanol/phosphate buffer solution (50/50, pH 8.0). The slow exchange between active iodine and tributyl stain was performed for 1 hour at room temperature. $1 \mathrm{~mL}$ of phosphate buffer and $2 \mathrm{~mL}$ of dichloromethane were then added and the iodinated-protected molecule contained in the organic phase was extracted. The aqueous phase contained radioactive iodides. The radiochemical purity was $\sim 70 \%$. The organic phase was evaporated and the pellet was resuspended in a mixture of phosphate buffer (0.01 M, pH 4)/acetonitrile (65/35) in which concentrated chlorhydric acid (10 M) was added to deprotect and regenerate the molecule functions. The mixture was then neutralized with sodium hydrohyde (NaOH, $0.1 \mathrm{~N})$. As the labeled compounds 4DIG, 4IEG and 6IPG were stable for over a month as determined by High Performance Liquid Chromatography studies (data not shown), ${ }^{125}$ I labeling (half-life $=60$ days) was used for those compounds instead of ${ }^{123}$ I to avoid repetitive labeling. 3IMG was labeled with iodine 123 (half-life $=13.3$ hours) because the stability of the compound did not exceed 48 hours.

\section{Biological procedures}

\section{In vivo studies: mouse biodistribution}

Female Swiss mice (average weight $20 \mathrm{~g}$ ) received $50 \mu \mathrm{L}$ of a solution of the iodinated glucose analogue $(7.4 \mathrm{kBq} / \mathrm{mouse})$ in isotonic $\mathrm{NaCl} 0.9 \%$. The animals were euthanized at 2, 5, 10, 15 and 60 minutes post-injection (pi). The heart, diaphragm, liver, lung, kidney, muscle, white fat and brain were excised, rinsed, weighed and counted in a scintillation y counter (Novelec). Tracer organ uptake was expressed as \% injected dose/g wet weight tissue (\% ID/g). Another group of mice was used to evaluate blood kinetics at the same time points. Tracer blood activity was expressed as $\%$ injected dose $/ \mathrm{mL}$ of blood $(\% \mathrm{ID} / \mathrm{mL})$.

\section{In vitro studies: human erythrocytes}

Human erythrocyte suspensions were prepared from whole blood according to the method of Levine et al. [22 ]. Erythrocytes were preincubated at $25^{\circ} \mathrm{C}$ or at $4^{\circ} \mathrm{C}$ to evaluate the effect of temperature on the cellular uptake of the tracers. The effect of the glucose transporter inhibitor cytochalasin B $(50 \mu \mathrm{M})$ on the cellular uptake kinetics of the iodinated glucose analogues was also studied by preincubating the erythrocyte suspension for $20 \mathrm{~min}$ at $4^{\circ} \mathrm{C}$ before adding the tracers. 42 nmoles of iodinated glucose analogue were then added to the cellular suspensions (10\% hematocrit). Tracer uptake was stopped after 1, 5, 15, and 60 min with an ice-cold solution of $\mathrm{HgCl}$ ${ }_{2}(2 \mathrm{mM}) / \mathrm{KI}(1.25 \mathrm{mM})$. The effect of preincubating the erythrocyte suspensions with increasing extracellular D-Glucose concentrations $(0$ $-20 \mathrm{mmol} / \mathrm{L}$ ) on the 1-min cellular uptake of iodinated glucose analogues was also evaluated. Finally, washout studies were performed by preincubating the erythrocytes with the iodinated glucose analogues (42 nmoles) for $60 \mathrm{~min}$ before rinsing and incubating the cells in a tracer-free medium in the absence or presence of cytochalasin B $(50 \mu \mathrm{M})$ for 30 and $60 \mathrm{~min}$. The use of erythrocyte suspensions for the study of new, radiolabeled glucose analogues has been previously validated with [ $\left.{ }^{14} \mathrm{C}\right]$-2-deoxy-D-glucose (2-DG) in our laboratory [23 ].

\section{Isolated rat adipocytes}

Isolated adipocytes were prepared as previously described [24 ] from epididymal fat pads of male Wistar rats (180-200 g). Briefly, epididymal fat pads were digested for $60 \mathrm{~min}$ at $37^{\circ} \mathrm{C}$ in a metabolic shaker using Krebs-Ringer bicarbonate $30 \mathrm{mM}$ HEPES buffer (KRBH, pH 7.4), containing $0.3 \mathrm{mM}$ glucose and 3\% BSA and supplemented with collagenase (1 mg/mL). The disrupted tissues were filtered through a $250 \mu \mathrm{M}$ nylon screen, and the cells were washed three times by flotation with KRBH buffer. Cellular suspensions (30\% adipocrit) were preincubated for $10 \mathrm{~min}$ at $37^{\circ} \mathrm{C}$ in $\mathrm{KRBH}, 3 \% \mathrm{BSA}$ without glucose. To study the uptake of iodinated glucose analogues, adipocytes $(100 \mu \mathrm{L})$ were preincubated in the absence or presence of $20 \mu \mathrm{M}$ cytochalasin $\mathrm{B}$ or $100 \mathrm{nM}$ insulin in $100 \mu \mathrm{L}$ KRBH, 3\% BSA without glucose for $30 \mathrm{~min}$ at $37^{\circ} \mathrm{C}$. Uptake was initiated by adding $50 \mu \mathrm{L}$ of the tracer ( $\left.25 \mathrm{nmoles}\right)$ and was stopped after $1,5,15$, and 30 $\min$. To study the effect of D-glucose concentration on the uptake of iodinated analogues of glucose, the adipocytes were preincubated for 5 minutes at $37^{\circ} \mathrm{C}$ with various concentrations of D-glucose $(0-20 \mathrm{mM})$ and the uptake was measured after 5 minutes. Tracer uptake was 
stopped by adding $10 \mu \mathrm{L}$ of cytochalasin B $(1 \mathrm{mM})$ and cells were immediately separated from the medium by centrifugation (10,000 rpm $\times 30 \mathrm{~s})$ of an aliquot of the cellular suspension $(200 \mu \mathrm{L})$ through $200 \mu \mathrm{L}$ dinonylphthalate. The tube was cut at the level of the oil phase and the uptake of iodinated glucose analogues by adipocytes was measured by gamma-well counting of the radioactivity (Novelec). The number of adipocytes was measured according to the method of Dole [25 ]. All experimental protocols on isolated rat adipocytes were validated by studying the effect of cytochalasin B, insulin, and increasing extracellular glucose concentrations on the cellular uptake of the gold-standard tracer for glucose uptake, $\left[{ }^{14} \mathrm{C}\right]$-2-deoxy-D-glucose (2-DG) (Fig. 2 and Table 2 ). 2-DG uptake increased over time to reach a value of $10 \mathrm{nmol} / 10^{6}$ cells after $30 \mathrm{~min}$. Insulin significantly stimulated tracer uptake from 5 to 30 min with a 3-fold increase at 30 min. Cytochalasine B inhibited 2-DG uptake by $80 \%$ at $15 \mathrm{~min}$. The D-glucose effect on 2-DG uptake was significant for concentrations above $10 \mathrm{mM}$ These results therefore validated the use of isolated rat adipocytes as an experimental model for the study of new radiolabeled glucose analogues.

\section{In vitro assessment of iodinated glucose analogue phosphorylation}

In vitro phosphorylation studies were performed on non-radioactive iodinated glucose analogues $(6 \mathrm{mM})$ in the presence of the hexokinase enzyme (100 units/L) and adenosine 5'-triphosphate (ATP, $0.1 \mathrm{mM}$ ) in the kit medium at room temperature. After 30 and 60 min, High-Performance Liquid Chromatography (HPLC, $\lambda=254 \mathrm{~nm}$ ) was performed to quantify the disappearance of ATP molecules, reflective of the glucose analogue's phosphorylation state. Positive controls were performed using D-glucose (6 mM) and showed near-complete disappearance of ATP.

\section{Statistical analysis}

Results are presented as mean \pm standard deviation. Comparisons were performed using unpaired Students't test.

\section{Results}

\section{In vivo studies: mouse biodistribution}

(Table 1 ) Myocardial activity of 3IMG was approximately 5\% ID/g at $2 \mathrm{~min}$ pi but decreased over time in the myocardium as well as in other organs. 4DIG activity was stable for up to $10 \mathrm{~min}$ in the myocardium and $15 \mathrm{~min}$ in skeletal muscle $(2.75 \pm 0.40 \% \mathrm{ID} / \mathrm{g}$ at $2 \mathrm{~min}$ pi and $2.73 \pm 0.30 \% \mathrm{ID} / \mathrm{g}$ at $15 \mathrm{~min}$ pi in skeletal muscle for example, NS). In the brain, 4DIG activity was low but increased between 2 and $15 \mathrm{~min}$ pi $(\mathrm{p}<0.001)$. The most part of 4IEG and 6IPG radioactivity was found in kidneys at $2 \mathrm{~min}$ pi $(33.6 \pm 2.4 \%$ ID/g for 4 IEG and $70.58 \pm 23.46 \%$ ID for $6 \mathrm{IPG}$ ) with almost no brain uptake. In summary, kidneys were the major organ of excretion for all iodinated glucose analogues. In addition, no major specific organ accumulation was observed with all tracers tested.

\section{In vitro studies: human erythrocytes}

\section{Uptake kinetics}

Uptakes of 3IMG and 4DIG by erythrocytes at $25^{\circ} \mathrm{C}$ strongly increased from 1 to $5 \mathrm{~min}(\mathrm{p}<0.001)$ and then remained stable until 60 min (Fig. 3a, 3b ). Uptakes of 4IEG and 6IPG at $25^{\circ} \mathrm{C}$ were low but also increased between 1 and $60 \mathrm{~min}$ (p < 0.001) (Fig. 3c, 3d ). A significant decrease in the cellular uptake of all iodinated glucose analogues was observed when decreasing the temperature at which the experiments were performed from 25 to $4^{\circ} \mathrm{C}$. Cytochalasin B had no effect on 3IMG uptake, with the exception of 15-min time point when a 55\% inhibition was observed ( $\mathrm{p}<0.01$ ). This inhibitor reduced 4DIG uptake from 5 min to $60 \mathrm{~min}(\mathrm{p}<0.05)$. However, this inhibition remained inferior to 50\%. Cytochalasin B had no effect on 4IEG and 6IPG uptake, except at 60 min for 6IPG (Fig. 3d, p<0.01).

\section{Competition with D-glucose (Table 2 )}

A preincubation with increasing concentrations of D-glucose from 2.5 to $20 \mathrm{mM}$ significantly decreased 3IMG uptake (p $<0.001)$. Likewise, 4DIG uptake was partially decreased in the presence of D-glucose at 2.5, 5 and $10 \mathrm{mM}(\mathrm{p}<0.05)$. On the other hand, increasing the extracellular concentration of D-glucose did not affect 4IEG or 6IPG uptake.

\section{Kinetics of washout (Table 3 )}

There was rapid washout of 3IMG from erythrocytes as only $5 \%$ of the initial radioactivity remained after 1 min. Cytochalasin B significantly slowed down 3IMG washout between $5 \mathrm{~min}$ and $30 \mathrm{~min}(\mathrm{p}<0.001)$. The washout of 4DIG was rapid as well, as only $8 \%$ of the initial radioactivity remained associated to the cells after $1 \mathrm{~min}$. Cytochalasin B decreased this washout as approximately $50 \%$ of the initial radioactivity still remained in the erythrocytes after $15 \mathrm{~min}(\mathrm{p}<0.001$ versus no cytochalasine $\mathrm{B}$ at the same time point). The washout of 4IEG was slower than that of 3IMG and 4DIG. Cellular tracer activity was still $50 \%$ of the initial value after 30 min of washout. Cytochalasin B had no effect on 4IEG washout from erythrocytes. The washout of 6IPG was slow, as $30 \%$ of the initial radioactivity remained associated to the cells after 1 min. Cytochalasin $B$ had no significant effect.

\section{Isolated Rat Adipocytes}


Uptake of the four iodinated analogues at $37^{\circ} \mathrm{C}$ by adipocytes remained relatively stable between 1 and 30 min (Fig. 4 ). 3IMG uptake was low ( 2 nmoles $/ 10^{6}$ cells). On the other hand, 4DIG and 4IEG uptakes represented $\sim 5 \mathrm{nmoles} / 10^{6}$ cells, a value similar to that observed with 2-DG between 5 and 15 min (Fig. 2 ). 6IPG uptake was the greatest with $\sim 10$ nmoles $/ 10^{6}$ cells. 4DIG uptake was increased by $30 \%$ at $30 \mathrm{~min}$ in the presence of insulin (p < 0.05) (Fig. 4b ). Cytochalasin B had significant effects on 4DIG uptake at $15 \mathrm{~min}$ (p < 0.05), on 4IEG uptake at $5 \mathrm{~min}(\mathrm{p}<0.05)$ and on 6IPG uptake at $5 \mathrm{~min}(\mathrm{p}<0.01)$ and $15 \mathrm{~min}(\mathrm{p}<0.05)$. However, these effects were lower than those observed on 2-DG uptake (Fig. 2 ).

\section{Competition with D-glucose}

An increase of D-glucose concentration in suspension medium from 2.5 to $20 \mathrm{mM}$ had no effect on the uptake of the four iodinated analogues on adipocytes (Table 2 ).

\section{In vitro assessment of iodinated glucose analogue phosphorylation}

No decrease in ATP concentration was observed for all four iodinated glucose analogues after 30 and 60 min of incubation in the presence of hexokinase, indicating no phosphorylation of the compounds.

\section{Discussion}

The aim of this study was to assess the biological behavior of new iodinated glucose analogues proposed as tracers of glucose uptake in vivo. This work is the final part of a systematic study initiated a few years ago, which consisted in adding an iodine atom on all free positions of the glucose molecule and in evaluating the potential interaction of these new molecules with glucose transporters on different biological models $[14,23$ ]. A radiolabeled glucose analogue iodinated on position 6 has previously been shown to interact with the glucose transporter, in a manner similar to that of the tracer of glucose transport 3-O-methyl-D-glucose (3-OMG) [11 ]. But none of the tracers evaluated so far behaved as a tracer of glucose uptake $[9,10,11,12,13,14,15,16,17]$. However, all labeling options for the glucose molecule had not been evaluated yet. New chemical synthesis strategies have been proposed, which enabled the labeling on the positions 3, 4, and 6 of the glucose molecules. The specific aim of this study was therefore to evaluate the biological behavior of four new glucose analogues iodinated in position 3, 4 and 6 and proposed as tracers of glucose uptake equivalent to 2-Deoxy-D-Glucose (2-DG). To be tracers of cellular glucose uptake, these iodinated glucose analogues should present several performance characteristics such as tissue selectivity, high cellular uptake, good affinity for the GLUTs, strong retention, and good interaction with hexokinase. As previously described by Henry et al. [23 ], the reference tracer of glucose uptake 2DG presented all these performance characteristics. The tracer was selective for the heart with myocardial uptake reaching $22 \% \mathrm{ID} / \mathrm{g}$ at $15 \mathrm{~min}$ pi [23 ]. In human erythrocytes, 2DG uptake increased linearly between 1 and $60 \mathrm{~min}$ at $25^{\circ} \mathrm{C}$. Cytochalasine B induced a 74\% inhibition of tracer uptake at 60 min and increasing the extracellular concentration of D-glucose was associated with a progressive decrease in 2DG uptake, demonstrating that 2DG cellular uptake was mediated by GLUTs. Finally, 2DG remained within the cell after being phosphorylated by hexokinase [26 ]. To study the effect of insulin, which stimulates glucose transport, the in vitro model of rat adipocytes in suspension was used in the present study. 2DG uptake was increased by 3-fold in the presence of insulin in this model (Fig. 2 ). The new iodinated glucose analogues should therefore present the same behavior if they are tracers of glucose uptake.

\section{Analogue iodinated in position 3 of the glucose molecule}

None of the glucose analogues iodinated in position 3 proposed so far has shown a biological behavior similar to that of 2-DG [9, 12 , $13,14,27,28$ ]. However, 3-OMG is methylated in position 3 and is still recognized by the glucose transporter. This position was therefore interesting for adding an iodine atom considering that the steric hinderance of this radioisotope is similar to that of a methyl group. Bessel et al. [29 ] have shown that the hydroxyl group in position 3 of the glucose molecule is necessary for the recognition of glucose by hexokinase. 3-OMG is therefore not a substrate for hexokinase due to the presence of a methyl group on carbon 3 and is not trapped in the cells. The glucose analogue 3IMG evaluated in the present study was then synthesized with a free hydroxyl group on carbon 3 (Fig. 1a ). 3IMG therefore potentially retains the capability to interact with hexokinase and to be trapped in the cell. Stabilization of the iodine atom on the glucose molecule was performed by using an iodomethyl group. After 3IMG radiolabeling through isotopic exchange, HPLC analysis showed a chemical stability exceeding 24 hours. Two min after in vivo injection in the mouse, high 3IMG kidney activity was observed, and tracer organ activity decreased in all organs over time without demonstrating any tissue selectivity. In erythrocytes, a rapid clearance of the molecule was observed. The effects of temperature, cytochalasin B, insulin and D-glucose were variable and not easily interpretable, demonstrating no clear interaction of the molecule with glucose transporters. Moreover, the in vitro phosphorylation assay indicated that 3IMG is not a substrate for hexokinase. These results suggest that 3IMG is not an iodinated analogue of 2-DG.

\section{Analogues iodinated in position 4 of the glucose molecule}

Only two glucose analogues iodinated in position 4 have been described so far: 4-O-(E)-3-iodopropen-2-yl-D-glucose and 4-O-(E)-3-iodopropen-2-yl-2-deoxy-D-glucose [10, 30 ]. No biological studies have been performed using these molecules. Glucose 
analogues iodinated in position 4 are potentially interesting because the $\mathrm{C} 4$ carbon does not play a major role in the recognition mechanism for the uptake of glucose into the cell via the glucose transporter, but is important in the recognition mechanism for its exit [31 ]. Thus, glucose analogues with voluminous groups in position 4 such as 4,6-O-ethyliden-D-glucose or 4-O-propyl-D-glucose have a good affinity for glucose transporters [32 ]. On the other hand, the glucose analogue modified in position 4, 4-deoxy-4-fluoro-D-glucose, is phosphorylated at a very slow rate by hexokinase [29 ]. Nevertheless, this radiolabeling position has not been extensively studied. Two glucose analogues have been studied in the present work: 4DIG, in which the hydroxyl group in position 4 of the glucose molecule was replaced by iodine, and 4IEG, which differs more from the original glucose molecule than 4DIG on a structural point of view, but which is also probably more chemically stable than this latter compound [33 ]. 4DIG. In the mouse, cardiac radioactivity of 4DIG was approximately 6-7\% ID/g 10 min following intravenous injection. 4DIG skeletal muscle activity was stable ( $3 \%$ ID/g) and radioactivity in the brain increased slightly from 2 to 15 minutes. This in vivo behavior seemed to be interesting even if uptakes were slightly inferior to those of 2-DG [23 ]. In human erythrocytes, temperature decreased 4DIG uptake by 50 to $60 \%$. A decrease in temperature reduces membrane fluidity and consequently decreases the simple and facilitated diffusion of a given molecule. This result suggests that 4DIG interacted with cytoplasmic membranes. Cytochalasin B decreased 4DIG uptake by 30 to $40 \%$ at 5 minutes, while a $90 \%$ decreased of 2-DG uptake was observed at 5 minute [23 ]. On isolated rat adipocytes, the only significant effect of cytochalasin B was observed at 15 minutes. On the other hand, cytochalasin B markedly inhibited the cellular washout of 4DIG from erythrocytes as $47 \%$ of the initial activity remained after a $15-\mathrm{min}$ washout when the glucose transporter inhibitor was present as opposed to $4 \%$ in its absence. These results were very different from those observed with 2-DG, which was trapped in the cells. However, they indicated that 4DIG probably interacts with glucose transporters. The different concentrations of D-glucose and insulin had only little effect on 4DIG uptake. The phosphorylation study showed that this molecule was not a substrate for hexokinase in vitro. The experimental results obtained with 4DIG indicated an interaction between 4DIG and glucose transporters during the cellular washout of the tracer. However, the cellular uptake of 4DIG does not occur through glucose transporters and the molecule is not phosphorylated. Therefore, this tracer cannot be considered as an equivalent of 2-DG. 4IEG. Biodistribution of 4IEG in the mouse showed high kidney uptake of the tracer at 2 minutes ( 35\% ID/g). In all organs, the clearance was rapid with the exception of the brain where nearly no tracer uptake was observed. In human erythrocytes, 4IEG uptake was low but increased with time and a decrease in temperature strongly decreased cellular tracer uptake by $80 \%$ at 60 minutes. This result suggests that 4IEG interacted with cytoplasmic membranes. Cytochalasin B had no major effect on 4IEG uptake and washout both in erythrocytes and adipocytes. Moreover, neither D-glucose nor insulin had a significant effect on 4IEG uptake indicating no affinity for the glucose transporter. Finally, this molecule was not phosphorylated in vitro by hexokinase. These results were very similar to those obtained by Henry et al. [23 ] with L- $\left[1-{ }^{14} \mathrm{C}\right]$-glucose, which enters into the cell via passive diffusion and is not further metabolized [18 ]. 4IEG does not present the performance characteristics expected and is therefore not a tracer of glucose uptake.

\section{Analogue iodinated in position 6 of the glucose molecule}

None of the glucose analogues iodinated in position 6 and previously described in the literature was phosphorylated by hexokinase, probably because of the substitution of the hydroxyl group by an iodine atom [11, 15, 20,34 ]. In this study, 6IPG was radiolabeled by substitution of the hydrogen on the C6 position with an iodine atom such as with 3IMG. The hydroxyl group in position 6 remained therefore potentially available for phosphorylation by the hexokinase enzyme. The aromatic cycle carrying the iodine atom is a very good stabilizing group, and two glucose analogues with substitution on the C6 position, 6-C-phenyl-[L and D]-glycerol-D-glucopyranose, have been described as inhibitors of the glucokinase enzyme [35 ]. An interaction between 6IPG and hexokinase is therefore possible. As observed for position 4, position 6 does not play a major role in the recognition of the glucose molecule by glucose transporters during cellular uptake. Indeed, 6-deoxy-6-iodo-D-glucose, in which the hydroxyl group in position 6 that has been substituted by an iodine atom, has shown a behavior similar to that of 3-OMG, the reference tracer of glucose transport [11 ]. Two minutes after injection in the mouse, 6IPG was mostly found in the kidney $(\sim 70 \% \mathrm{ID} / \mathrm{g})$. Tracer uptake by erythrocytes was slow and not markedly affected by temperature decrease. Cytochalasin B effect on 6IPG uptake by erythrocytes and adipocytes was also weak and the glucose transporter inhibitor did not inhibit the cellular washout of the tracer. Neither D-glucose nor insulin had an effect on the cellular uptake of this molecule, and 6IPG was not a substrate for hexokinase in vitro. Taken together, the results indicate that 6IPG does not interact with glucose transporters and is not phosphorylated. 6IPG cannot be considered as a tracer of glucose uptake.

The main findings of this study are summarized in the table below. 
Iodinated analogues

3IMG

4DIG

4IEG

6IPG
Uptake rate

Transporter selectivity

low none

cellular washout only

none

low

low

none
Retention

none

none

slow washout

none

none

none

none

none

none

none

none 


\section{Conclusion}

The aim of this study was to assess the biological behavior of new radiolabeled glucose analogues iodinated in position 3, 4 and 6 and proposed as equivalents of 2-DG, the reference tracer of glucose transport and phosphorylation. None of these tracers displayed the required biological characteristics of a tracer of glucose uptake. The present study represented the last part of the systematic study consisting in adding an iodine atom on all free positions of the glucose molecule. None of the molecule tested showed an affinity for the hexokinase and therefore can be proposed as a true glucose tracer. However, 6-deoxy-6-iodo-D-glucose (6DIG) has previously been validated as a tracer of glucose transport in vitro [11 ]. In vivo studies aimed at demonstrating the usefulness of this tracer are currently in progress in our laboratory.

\section{References:}

- 1. Schelbert HR, Buxton D . Insights into coronary disease gained from metabolic imaging . Circulation . 1988; $78: 496-505$

- 2. Garvey WT , Birnbaum MJ . Editor: Ferrannini E. Cellular insulin action and insulin resistance . Bailleiere's Clinical Endocrinology and Metabolism: Insulin resistance and disease . London Bailleiere Tindall ; $1994 ; 785$ - 873

- 3. Rigo P, Paulus P, Kaschten BJ , Hustinx R , Bury T, Jerusalem G, Benoit T, Foidart-Willems J . Oncological applications of positron emission tomography with fluorine-18 fiuorodeoxyglucose . Eur J Nucl Med . 1996; $23: 1641$ - 74

- 4. Weber MJ, Salter DW, McNair TE . Editor: Arnott MS, Van Eys J, Wang Y-M . Increased glucose transport in malignant cells: analysis of its molecular basis . Molecular interrelations of nutrition and cancer. New York Raven Press ; $1982 ; 183$ - 90

- 5. Opie LH , Hesse B . Radionuclide tracers in the evaluation of resting myocardial ischaemia and viability . Eur J Nucl Med . $1997 ; 24: 1183$ - 93

- 6 . Ishii K, Sasaki M, Kitagaki H, Yamaji S, Sakamoto S, Matsuda K, Mori E. Reduction of cerebellar glucose metabolism in advanced Alzheimer's disease . J Nucl Med . $1997 ; 38: 925-28$

- 7. Phelps ME, Huang SC, Hoffman EJ, Selin C, Sokoloff L, Kuhl DE . Tomographic measurement of local cerebral glucose metabolic rate in humans with (F-18)2-fluoro-2-deoxy-D-glucose: Validation of method . Ann Neurol . 1979; 6:371 - 88

- 8. Delbeke D . Oncological applications of FDG PET imaging: brain tumors, colorectal cancer lymphoma and melanoma . J Nucl Med . 1999 ; 40 : 591 - 603

- 9. Goodman MM , Kabalka GW , Waterhouse RN , Daniel GB . Synthesis of iodine-123 labeled 3-0-(E)-3-iodopropen-2-yl-D-glucose: a potential new agent for the assessment of glucose transport into the brain and heart using SPECT . J Labelled Compd Radiopharm . $1991 ; 30: 278-79$

- 10 . Goodman MM, Kabalka GW , Meng X . Synthesis of iodine-123 labeled 4-0-(E)-3-iodopropen-2-yl-2-deoxy-D-glucose and 4-0-(E)-3-iodopropen-2-yl-D-glucose as potential brain and heart imaging agents . J Labelled Compd Radiopharm . $1991 ; 30: 280-82$

- 11 . Henry C , Koumanov F, Ghezzi C , Morin C , Mathieu J-P , Vidal M , de Leiris J , Comet M . [ ${ }^{123}$ I]-6-deoxy-6-iodo-D-glucose (6DIG), a potential tracer of glucose transport . Nucl Med Biol . $1997 ; 24: 527-34$

- 12. Kloster G, Laufer P, Stöcklin G . D-glucose derivatives labeled with ${ }^{75,77}$ Br- and ${ }^{123}$ I . J Labelled Compd Radiopharm . 1983 ; 20 : 391 - 415

- 13 . Kloster G, Laufer P, Wutz W, Stöcklin G. ${ }^{75,77}$ Br- and ${ }^{123}$ I-analogues of D-glucose as potential tracers for glucose utilization in heart and brain . Eur J Nucl Med . $1983 ; 8: 237-41$

- 14. Koumanov F, Henry C, Ghezzi C, Bignan G, Morin C, Mathieu J-P, Hamant S, Vidal M, de Leiris J, Comet M . Biological studies of analogues of glucose iodinated in positions 1, 2, or 3. Nucl Med Biol . 1996;23:53-60

- 15. Koumanov F, Henry C, Ghezzi C, Mathieu J-P, Morin C, Vidal M, de Leiris J , Comet M . Biological valuation of two anomeric glucose analogues iodinated in position 6. Nucl Med Biol . $1997 ; 24: 519-25$

- 16 . Lutz T, Dougan H, Rihela T, Hudon M, Cohen P, Jamieson WRE, Lyster DM . The effect of iodine position on uptake in the mouse using an isomeric series of 2-deoxy-2-0-([ ${ }^{123}$ I]-iodobenzyl)glucose . J Labelled Compd Radiopharm . $1991 ; 29: 535$ - 45

- 17 . Magata Y, Saji H, Ohmomo Y, Tanaka C, Konishi J, Yokoyama A . Development of a novel radioiodinated glucose derivative with interaction to hexokinase . J Labelled Compd Radiopharm . $1992 ; 31: 317-28$

- 18. LeFevre PG . Sugar transport in the red blood cell: structure - activity relationships in substrates and antagonists . Physiol Rev . $1961 ; 13: 39$ - 70

- 19. Abbadi M, Mathieu J-P, Morin C . A synthesis of 4-deoxy-D-glucose suitable for radiolabeling . J Labelled Compd Radiopharm . $1997 ; 39$ : 487 - 92

- 20 . Morin C, Ogier L. Synthesis of 4-O- and 6-O-(2'-iodoethyl)-D-glucose . Carbohydr Res . $1998 ; 310: 277$ - 82

- 21 . Mathieu J-P, Riche F, Coornaert S, Bardy A, Busquet G, Godart J, Comet M, Vidal M. Marquage d'acides gras en position $\omega$ par les isotopes de l'iode . J Biophys Med Nucl . $1982 ; 6: 233-37$

- 22. Levine M, Levine S, Jones MN. The effect of temperature on the competitive inhibition of sorbose transfer in human erythrocytes by glucose . Biochim Biophys Acta Sci. $1971 ; 225: 291-300$

- 23. Henry C, Koumanov F, Ghezzi C, Mathieu J-P, Hamant S, de Leiris J, Comet M . Experimental models, protocols and reference values for evaluation of iodinated analogues of glucose . Nucl Med Biol . $1995 ; 22: 875-85$

- 24 . Rodbell M. Metabolism of isolated fat cells. I. Effects of hormones on glucose metabolism and lipolysis . J Biol Chem . $1964 ; 239$ : 375 - 80

- 25. Dole VP. The fatty acid pool in adipose tissue. J Biol Chem . $1961 ; 236: 3121-24$

- 26. Sols A, Crane RK . Substrate specificity of brain hexokinase . J Biol Chem . $1954 ; 210: 581$ - 95

- 27 . Goodman MM , Waterhouse RN , Kabalka GW , Knapp FF. Synthesis and biological evaluation of 3-C((E)-2-[ ${ }^{125}$ I]iodoethenyl)-D-allose: A new strategy for preparation of in vivo stable radioiodinated carbohydrates. NUC Compact . $1990 ; 21: 64$ - 9

- 28 . Kloster G, Laufer P, Wutz W, Stöcklin G. ${ }^{75}$ Br- and ${ }^{123}$ I-analogues of D-glucose as potential radiopharmaceuticals . J Labelled Compd Radiopharm . 1981 ; 19 : 1626 $-28$

- 29. Bessell EM , Foster AB , Westwood JH . The use of deoxyfluoro-D-glucopyranoses and related compounds in a study of yeast hexokinase specificity . Biochem J . 1972 ; $128: 199-204$

- 30 . Goodman MM, Kabalka GW , Meng X, Daniel GB , Longford CPD . Synthesis and evaluation of radioiodinated 4-0-(E)-3-iodopropen-2-yl-D-glucose and derivatives as potential heart imaging agents. J Nucl Med . $1990 ; 31$ - Abstract 900

- 31. Barnett JEG, Holman GD, Chalkley RA, Munday KA . Evidence for two asymmetric conformational states in the human erythrocyte sugar-transport system . Biochem J $1975 ; 145: 417-29$

- 32. Barnett JEG, Holman GD, Munday KA . Structural requirements for binding to the sugar-transport system of the human erythrocyte . Biochem J . 1973 ; 131 : 211 - 21

- 33 . Hamant S, Mathieu J-P, Morin C, Trimcev I, Vidal M. The $\beta$-iodoethoxyl group: a stable unit for radioiodination . Bioorg Med Chem Letters . 1994 ; 4 : 1687 - 90

- 34 . Flanagan R, Lentle BC, Wiebe LI, Helus F, Maier-Borst W. Synthesis of 6-radioiodo-6-deoxy-D-galactose, and its tissue distribution and excretion in rats . Radiopharm (Proc Int Symp, 2nd) . $1979 ; 2: 119$ - 28

- 35 . Blériot Y, Veighey CR, Smelt KH, Cadefau J, Stalmans W, Biggadike K, Lane AL, Muller M, Watkin DJ , Fleet GWJ . The first example of a 6-C-aryl-D-glucose: inhibition of glucokinase. Tetrahedron: Asymm . 1996; $7: 2761-72$ 
Figure 1

Structure of the iodinated glucose analogues: (a) 3IMG, (b) 4DIG, (c) 4IEG and (d) 6IPG.
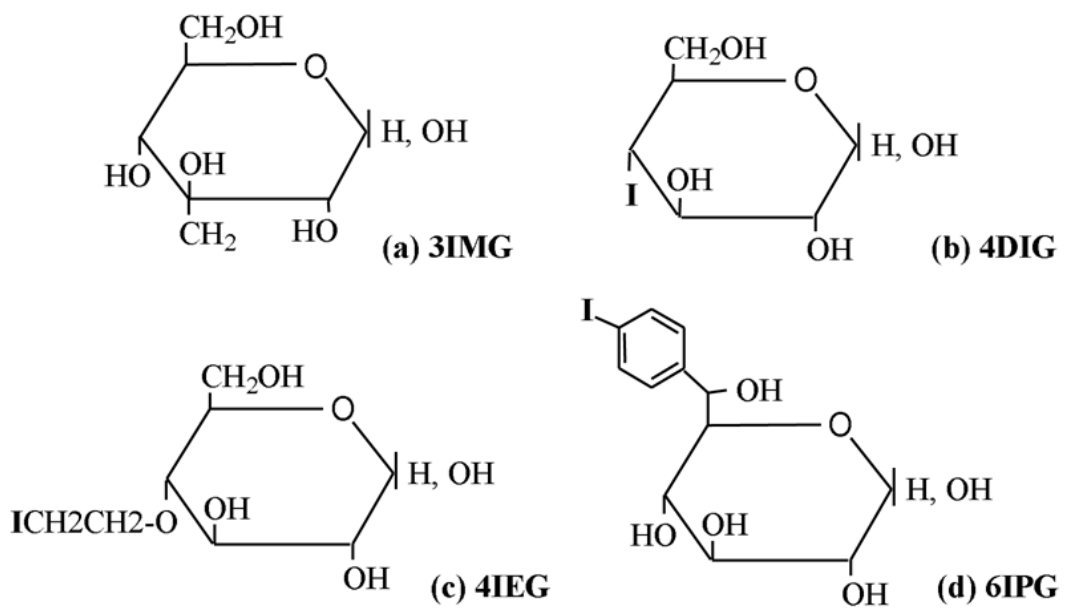

\section{Figure 2}

Time course of 2-deoxy-D-glucose (2DG) uptake by adipocytes isolated from rats in suspension: control: $\square$, insulin: • and cytochalasin B (20 $\mu \mathrm{M})$ : grey bars. Mean $\pm \mathrm{SD}\left(\mathrm{n}=3\right.$ for each time point). Comparison with control values: ${ }^{*} \mathrm{p}<0.05,{ }^{* *} \mathrm{p}<0.01$ and ${ }^{* * *} \mathrm{p}<0.001$.

2DGi uptake (nmol/ $10^{6}$ cells)

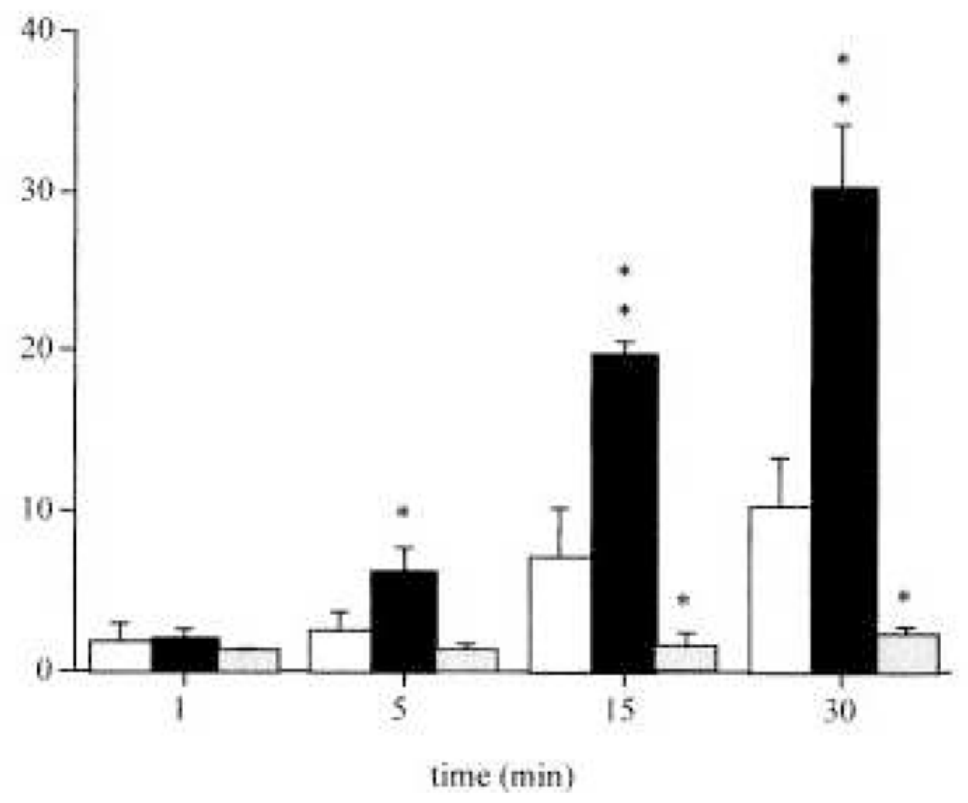


Figure 3

Time course of the iodinated glucose analogues uptake by human erythrocytes in suspension, (a) 3IMG, (b) 4DIG, (c) 4IEG and (d) 6IPG. $\square$ : $25^{\circ} \mathrm{C},-4^{\circ} \mathrm{C}$ and grey bars: $4^{\circ} \mathrm{C}+$ cytochalasin $\mathrm{B}(50 \mu \mathrm{M})$. Mean $\pm \mathrm{SD}(\mathrm{n}=3$ for each time point $)$. Comparison with control values: ${ }^{*} \mathrm{p}<0.05$, * ${ }^{*} \mathrm{p}<0.01$ and ${ }^{* * *} \mathrm{p}<0.001$.

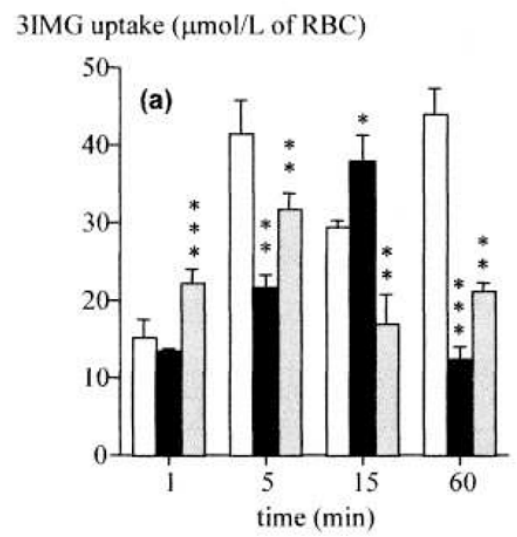

$$
\text { 4DIG uptake }(\mathrm{mmol} / \mathrm{L} \text { of } \mathrm{RBC})
$$

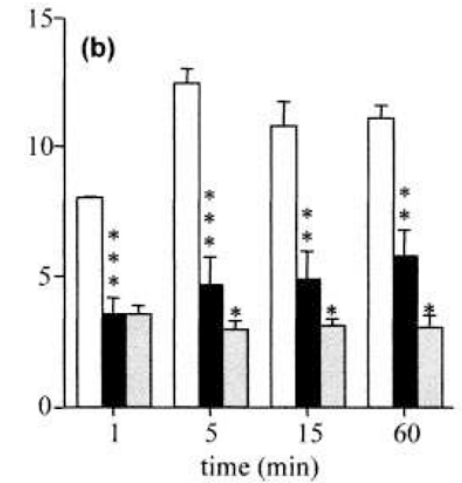

4IEG uptake $(\mu \mathrm{mol} / \mathrm{L}$ of $\mathrm{RBC})$
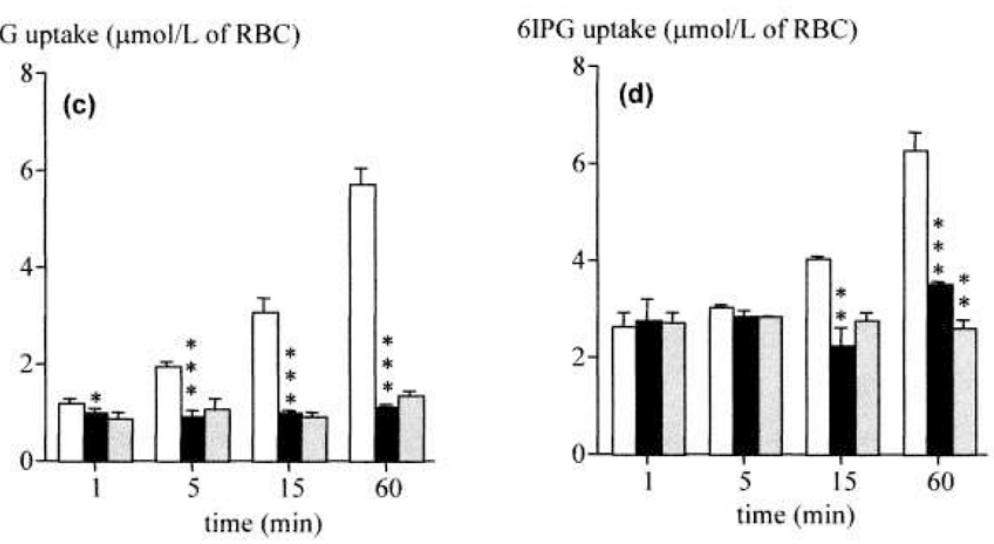

\section{Figure 4}

Time course of the iodinated glucose analogues uptake by adipocytes isolated from rats in suspension, (a) 3IMG, (b) 4DIG, (c) 4IEG and (d) 6IPG. $\square$ : control, $\mathbf{-}$ : insulin and grey bars: cytochalasin $(20 \mu \mathrm{M})$. Mean $\pm \mathrm{SD}\left(\mathrm{n}=3\right.$ for each time point). Comparison with control values: ${ }^{*} \mathrm{p}<$ $0.05,{ }^{* *} \mathrm{p}<0.01$ and ${ }^{* * *} \mathrm{p}<0.001$.
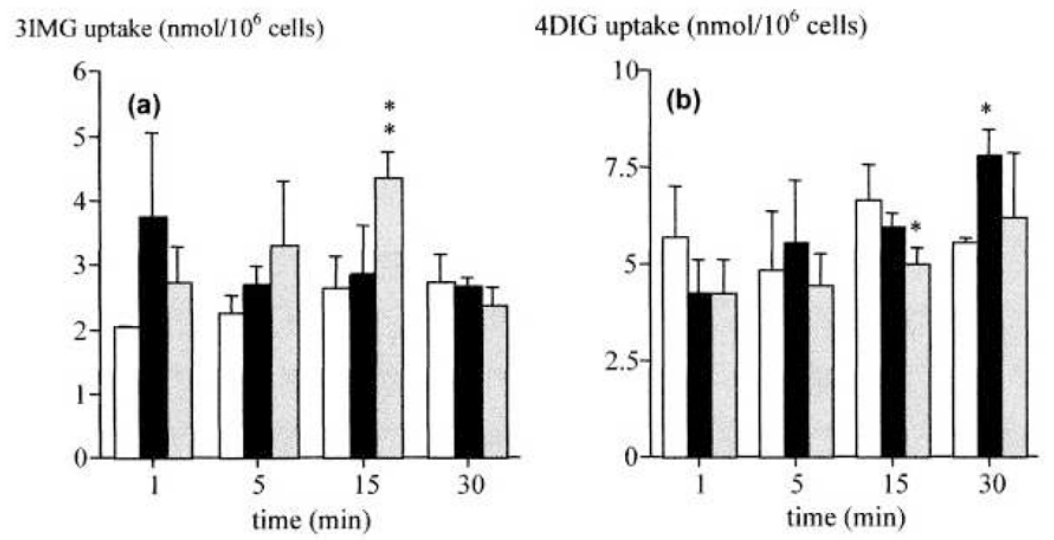

4IEG uptake $\left(\mathrm{nmol} / 10^{6}\right.$ cells)

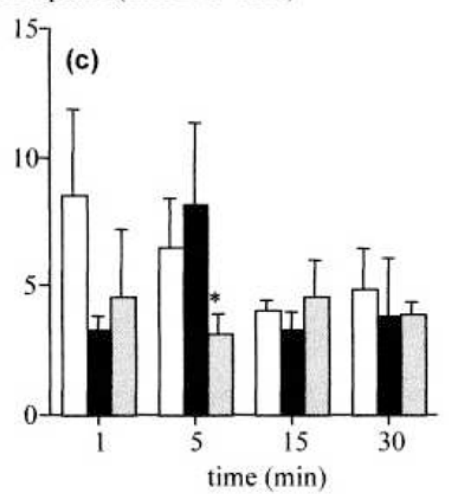

6IPG uptake ( $\mathrm{nmol} / 10^{6}$ cells)

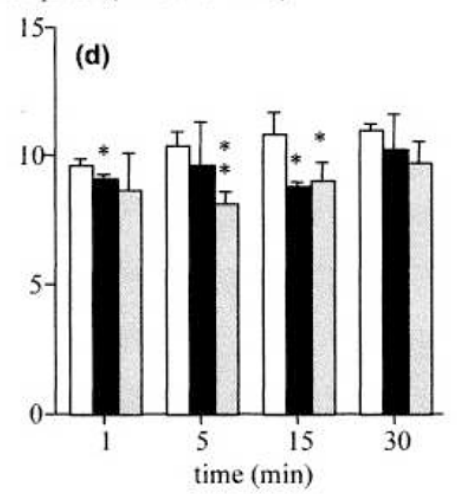


TABLE 1

Biodistribution in the mouse of the iodinated glucose analogues.

1a. Biodistribution of 3IMG

\begin{tabular}{|c|c|c|c|c|c|c|c|}
\hline Time (min) & Heart & Muscle & Brain & Lung & Liver & Kidney & Blood \\
\hline 2 & $5.20 \pm 0.02$ & $2.34 \pm 0.11$ & $0.64 \pm 0.18$ & $8.09 \pm 0.86$ & $9.52 \pm 0.81$ & $20.24 \pm 0.79$ & $14.19 \pm 1.34$ \\
\hline 5 & $4.42 \pm 0.29^{*}$ & $1.98 \pm 0.25$ & $0.48 \pm 0.06$ & $6.81 \pm 0.67$ & $6.35 \pm 0.05^{* *}$ & $11.84 \pm 1.45^{* * *}$ & $11.22 \pm 0.96^{*}$ \\
\hline 10 & $3.68 \pm 0.50^{* *}$ & $1.81 \pm 0.05$ & $0.62 \pm 0.20$ & $6.11 \pm 0.51^{*}$ & $4.23 \pm 0.14^{\star \star *}$ & $7.67 \pm 1.25^{* \star *}$ & $9.54 \pm 1.38^{*}$ \\
\hline 15 & $2.76 \pm 0.14^{* * *}$ & $1.94 \pm 0.29^{* * *}$ & $0.94 \pm 0.78$ & $5.66 \pm 0.48^{*}$ & $3.72 \pm 0.27^{* * *}$ & $7.30 \pm 1.31^{* * *}$ & $8.56 \pm 1.26^{* *}$ \\
\hline \multicolumn{8}{|c|}{ 1b. Biodistribution of 4DIG } \\
\hline Time (min) & Heart & Muscle & Brain & Lung & Liver & Kidney & Blood \\
\hline 2 & $6.62 \pm 0.76$ & $2.75 \pm 0.40$ & $1.25 \pm 0.23$ & $7.44 \pm 0.84$ & $11.10 \pm 0.25$ & $15.43 \pm 2.62$ & $9.39 \pm 1.59$ \\
\hline 5 & $6.37 \pm 0.39$ & $2.76 \pm 0.31$ & $1.54 \pm 0.09^{*}$ & $7.25 \pm 0.55$ & $8.79 \pm 0.42^{* * *}$ & $12.06 \pm 0.82^{*}$ & $9.89 \pm 1.52$ \\
\hline 10 & $6.07 \pm 1.06$ & $2.62 \pm 0.52$ & $1.72 \pm 0.38^{*}$ & $6.39 \pm 0.72$ & $7.11 \pm 0.93^{* * *}$ & $9.11 \pm 1.39^{* * *}$ & $6.97 \pm 0.60^{* *}$ \\
\hline 15 & $4.88 \pm 0.52^{* *}$ & $2.73 \pm 0.30$ & $2.14 \pm 0.22^{* * *}$ & $5.50 \pm 0.39^{* * *}$ & $6.08 \pm 0.70^{* * *}$ & $7.83 \pm 0.42^{* * *}$ & $5.63 \pm 1.20^{* *}$ \\
\hline \multicolumn{8}{|c|}{ 1c. Biodistribution of 4IEG } \\
\hline Time (min) & Heart & Muscle & Brain & Lung & Liver & Kidney & Blood \\
\hline 2 & $6.56 \pm 1.72$ & $3.81 \pm 0.22$ & $0.39 \pm 0.08$ & $9.38 \pm 1.58$ & $12.95 \pm 1.56$ & $33.68 \pm 2.42$ & $14.05 \pm 1.74$ \\
\hline 5 & $6.79 \pm 0.44$ & $3.94 \pm 0.41$ & $0.48 \pm 0.07$ & $9.01 \pm 0.38$ & $12.04 \pm 0.92$ & $28.04 \pm 7.38$ & $12.93 \pm 1.59$ \\
\hline 10 & $5.15 \pm 0.47$ & $3.68 \pm 0.44$ & $0.36 \pm 0.04$ & $5.39 \pm 0.58^{*}$ & $6.87 \pm 1.10^{* *}$ & $13.21 \pm 0.24^{\star \star *}$ & $6.21 \pm 0.60^{* *}$ \\
\hline 15 & $3.30 \pm 0.39^{*}$ & $2.76 \pm 0.35^{*}$ & $0.27 \pm 0.06$ & $2.94 \pm 0.48^{* *}$ & $3.38 \pm 0.61^{* * *}$ & $6.99 \pm 0.43^{* * *}$ & $3.16 \pm 0.99^{* * *}$ \\
\hline \multicolumn{8}{|c|}{ 1d. Biodistribution of 6IPG } \\
\hline Time (min) & Heart & Muscle & Brain & Lung & Liver & Kidney & Blood \\
\hline 2 & $4.51 \pm 0.94$ & $2.23 \pm 0.49$ & $0.33 \pm 0.11$ & $8.73 \pm 1.07$ & $25.76 \pm 7.66$ & $70.58 \pm 23.46$ & $10.36 \pm 1.35$ \\
\hline 5 & $3.85 \pm 0.34$ & $2.20 \pm 0.20$ & $0.33 \pm 0.08$ & $7.45 \pm 0.78$ & $31.22 \pm 2.60$ & $37.91 \pm 2.91$ & $7.78 \pm 0.61^{*}$ \\
\hline 10 & $3.92 \pm 0.37$ & $2.13 \pm 0.26$ & $0.20 \pm 0.05$ & $7.12 \pm 0.84$ & $29.36 \pm 3.17$ & $28.35 \pm 3.72^{*}$ & $7.76 \pm 0.74^{*}$ \\
\hline 15 & $3.33 \pm 0.10$ & $1.84 \pm 0.18$ & $0.64 \pm 0.54$ & $5.21 \pm 0.48^{* *}$ & $22.21 \pm 2.85$ & $17.69 \pm 2.13^{*}$ & $6.50 \pm 0.08^{* *}$ \\
\hline
\end{tabular}

Results are expressed as \% of the injected dose per gram of organ (\% ID/g) or per millilitre of blood (\% ID/ml). Mean \pm SD ( $n=3$ ). Each value was compared to that obtained at 2 min pi ${ }_{* *}^{*} \mathrm{p}<0.05$

${ }_{* *} p<0.01$ and

$\mathrm{p}<0.001$. 
TABLE 2

Effects of D-glucose concentration on the uptake of the iodinated glucose analogues by human erythrocytes in suspension at $4{ }^{\circ} \mathrm{C}$ and in adipocytes isolated from rats at $37^{\circ} \mathrm{C}$.

\begin{tabular}{|c|c|c|c|c|c|c|}
\hline & D-glucose concentration $(\mathrm{mM})$ & $\mathbf{0}$ & 2.5 & 5 & 10 & 20 \\
\hline \multirow[t]{2}{*}{$2 \mathrm{DG}$} & RBC & $3.52 \pm 0.16$ & $2.76 \pm 0.05^{* *}$ & $2.46 \pm 0.30^{* * *}$ & $1.69 \pm 0.11^{* \star *}$ & $1.15 \pm 0.12^{* * *}$ \\
\hline & adipocytes & $3.02 \pm 0.58$ & $3.19 \pm 0.52$ & $3.18 \pm 0.25$ & $2.28 \pm 0.33^{\star * *}$ & $2.05 \pm 0.28^{*}$ \\
\hline \multirow[t]{2}{*}{ 3IMG } & $\mathrm{RBC}$ & $42.99 \pm 0.31$ & $22.88 \pm 1.25^{\star \star \star}$ & $22.21 \pm 0.95^{\star \star \star}$ & $14.99 \pm 0.56^{* * *}$ & $14.98 \pm 1.05^{* * *}$ \\
\hline & adipocytes & $3.45 \pm 1.75$ & $2.75 \pm 0.05$ & $3.56 \pm 0.75$ & $3.92 \pm 0.52$ & $3.47 \pm 0.38$ \\
\hline \multirow[t]{2}{*}{$4 \mathrm{DIG}$} & $\mathrm{RBC}$ & $4.66 \pm 0.36$ & $3.48 \pm 0.11^{*}$ & $3.55 \pm 0.26^{*}$ & $3.92 \pm 0.89^{*}$ & $4.08 \pm 0.41$ \\
\hline & adipocytes & $5.67 \pm 0.49$ & $6.54 \pm 1.41$ & $4.50 \pm 0.60$ & $5.47 \pm 1.24$ & $5.22 \pm 0.56$ \\
\hline \multirow[t]{2}{*}{ 4IEG } & $\mathrm{RBC}$ & $0.21 \pm 0.01$ & $0.20 \pm 0.01$ & $0.20 \pm 0.01$ & $0.20 \pm 0.01$ & $0.21 \pm 0.01$ \\
\hline & adipocytes & $2.57 \pm 0.32$ & $3.00 \pm 0.52$ & $2.08 \pm 0.24$ & $2.69 \pm 0.72$ & $2.32 \pm 0.28$ \\
\hline \multirow[t]{2}{*}{ 6IPG } & $\mathrm{RBC}$ & $2.94 \pm 0.02$ & $3.01 \pm 0.17$ & $3.05 \pm 0.06$ & $2.91 \pm 0.16$ & $2.97 \pm 0.12$ \\
\hline & adipocytes & $10.07 \pm 0.67$ & $10.10 \pm 0.25$ & $10.07 \pm 0.83$ & $9.92 \pm 0.35$ & $10.72 \pm 0.80$ \\
\hline
\end{tabular}

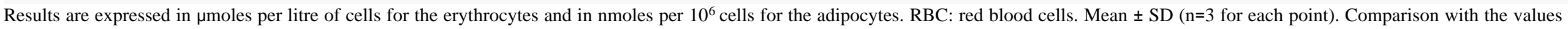
meseared in the absence of D-glucose:

${ }_{* *}^{*} \mathrm{p}<0.05$

${ }^{* *} \mathrm{P}<0.01$ and

$\mathrm{p}<0.001$.

$\mathrm{p}<0.001$.
Values for the uptake of 2-DG by human erythrocytes have been previously published by our laboratory [ $\left.{ }^{23}\right]$.

\section{TABLE 3}

Washout of the iodinated glucose analogues from human erythrocytes in the absence (-) or in the presence (+) of cytochalasin B.

\begin{tabular}{|c|c|c|c|c|c|}
\hline Time & $(\min )$ & 1 & 5 & 15 & 30 \\
\hline \multirow[t]{2}{*}{ 3IMG } & - & $4.86 \pm 0.52$ & $2.18 \pm 0.04$ & $1.67 \pm 0.17$ & $1.22 \pm 0.14$ \\
\hline & + & $4.97 \pm 0.10$ & $4.04 \pm 0.16^{* \star *}$ & $3.19 \pm 0.19^{* *}$ & $3.73 \pm 0.24^{* \star *}$ \\
\hline \multirow[t]{2}{*}{ 4DIG } & - & $8.55 \pm 0.78$ & $4.73 \pm 0.08$ & $3.95 \pm 0.46$ & ND \\
\hline & + & $58.55 \pm 0.74^{\star * \star}$ & $53.00 \pm 3.08^{* * *}$ & $47.01 \pm 1.07^{* \star *}$ & ND \\
\hline \multirow[t]{2}{*}{ 4IEG } & - & $82.87 \pm 3.40$ & $77.10 \pm 0.72$ & $68.33 \pm 1.11$ & $52.02 \pm 2.05$ \\
\hline & + & $88.43 \pm 0.09$ & $77.44 \pm 0.38$ & $74.32 \pm 0.87^{* *}$ & $54.57 \pm 1.67$ \\
\hline 6IPG & + & $32.81 \pm 0.89$ & $39.60 \pm 2.85$ & $40.28 \pm 1.25$ & $26.95 \pm 2.37$ \\
\hline
\end{tabular}

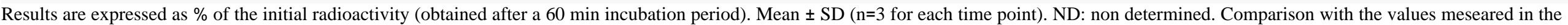
absence of cytochalasin $\mathrm{B}$.

${ }_{* *}^{*} \mathrm{p}<0.05$,

${ }_{* \star *}^{* *} \mathrm{p}<0.01$ and

$\mathrm{p}<0.001$. 